\title{
Massless Leggett Mode in Three-band Superconductors with Time-Reversal-Symmetry Breaking
}

\author{
Shi-Zeng Lin and Xiao $\mathrm{Hu}$ \\ WPI Center for Materials Nanoarchitectonics, National Institute for Materials Science, Tsukuba 305-0044, Japan
}

(Dated: November 5, 2018)

\begin{abstract}
The Leggett mode associated with out-of-phase oscillations of superconducting phase in multi-band superconductors usually is heavy due to the interband coupling, which makes its excitation and detection difficult. We report on the existence of a massless Leggett mode in three-band superconductors with time-reversal-symmetrybreaking (TRSB). The mass of the Leggett mode is small close to the TRSB transition and vanishes at the transition point, and thus locates within the smallest superconducting energy gap, which makes it stable and detectable. The mass of the Leggett mode can be measured by Raman spectroscopy. The thermodynamic consequences of this massless mode and possible realization in iron-based superconductors are also discussed.
\end{abstract}

PACS numbers: 74.20.-z, 03.75.Kk, 67.10.-j

Introduction - Spontaneous breaking of a continuous symmetry and the associated low-energy collective excitation govern the physical properties in many systems ranging from condensed matter physics to particle physics. Superconductivity emerging as the spontaneous breaking of the $U(1)$ gauge symmetry supports a massless excitation known as Bogoliubov-Anderson-Goldstone (BAG) boson[1, 2]. Coupled with electromagnetic field, the BAG boson becomes the massive plasma mode due to the Anderson-Higgs mechanism.

Because of the discoveries of $\mathrm{MgB}_{2}[3]$ and iron pnictides[4], it is now accepted that multi-component superconductors are ubiquitous. Multi-band superconductors are not straightforward extensions of the single-band counterpart, novel features arise instead[5-10]. A famous example is the Leggett mode (LM) in two-band superconductors associated with the collective oscillation of superconducting condensates between different bands, as schematically depicted in Fig. 1, with the mass proportional to the interband coupling. [5] In 2007, Blumberg et. al. reported the observation of the LM in $\mathrm{MgB}_{2}$ with the Raman spectroscopy[9]. The mass of the mode lies between the two superconducting energy gaps, consistent with the theoretical calculations[11]. The $\mathrm{LM}$ in $\mathrm{MgB}_{2}$ therefore decays into quasiparticle continuum associated with the band of smaller energy gap. The heavy $\mathrm{LM}$ in $\mathrm{MgB}_{2}$ has also been observed in point-contact transport measurements [12].

For iron-based superconductors, many studies have revealed the sign-reversal pairing symmetry between different bands $13-16]$. The system of more than three bands is somehow frustrated, and under appropriate conditions there may exist time-reversal-symmetry-breaking (TRSB) states even with conventional s-wave pairing symmetry, which involve non-trivial phase differences (i.e. $\delta \varphi \neq 0$ or $\pi$ ) among superconducting gaps[6, 17- 19]. With the new TRSB transition below $T_{c}$, the transition temperature of superconductivity, the spectrum of collective excitations and thus low-energy physical properties of the superconductors should be modified significantly. It was reported that the LM may exist below the two-particle continuum in iron-based superconductors under appropriate conditions, [20] and that the mass of the LM may be reduced in some dynamical classes of multiple interband Josephson coupling in three-band superconductors. [21]

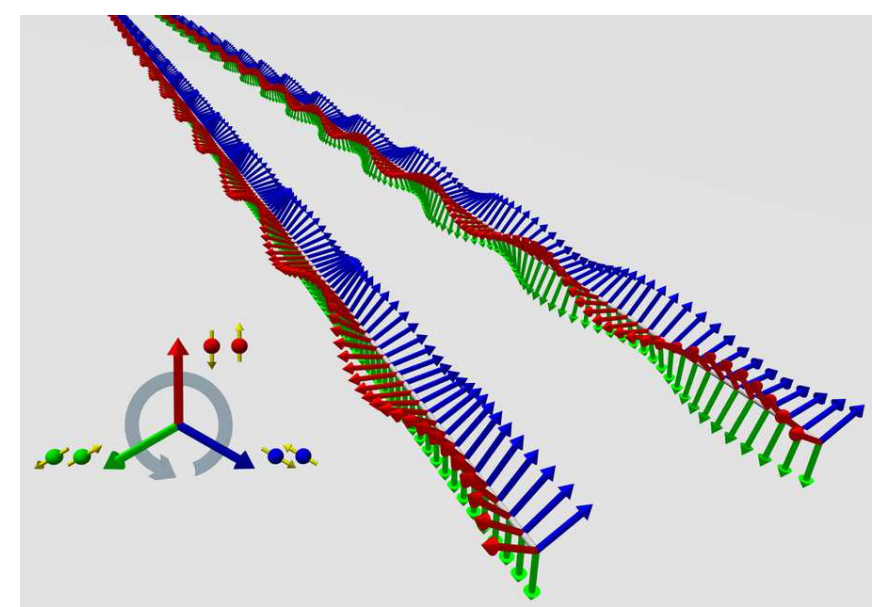

FIG. 1. Frustrated interband scatterings force Cooper pairs in different bands carry different phases, which results in interband Josephson currents. Two dynamical modes associated with superconducting phases in three-band superconductors: the LM, where one of the three phases stays unchanged while the other two oscillate out-ofphase, becomes massless at the TRSB transition (left), and the BAG mode, where all the three phases rotate in the same direction during the propagation of plasma wave in space (right).

We note that in another TRSB superconducting systems with mixed-symmetry order parameters with nodes such as $d+i s$, a massive LM in the TRSB state was found in Ref. [22]. With all these recent progresses in mind, we ask a question which is of fundamental interest: what are the effects of frustration on the LM and is it possible to have a massless LM mode?

In the present work, we demonstrate that the mass of the LM can be reduced significantly and even vanishes at TRSB transition upon sweeping interband coupling or density of states in multi-band superconductors. It is shown that the LM of vanishing mass can be detected by Raman scattering, which also serves as a smoking gun evidence for the TRSB transition. The appearance of massless excitation modifies superconductivity properties qualitatively, such as the power-law dependence of the specific heat $(\mathrm{SH})$ on temperature instead of the conventionally exponential one for full-gapped systems. It is found that several recent experiments on iron-based su- 
perconductors can be explained by the existence of massless LM.

Leggett mode - The Hamiltonian for three separate pieces of the isotropic Fermi surface can be written as

$$
\begin{aligned}
& H=\sum_{l, \sigma} \int d^{3} r \psi_{l \sigma}^{\dagger}(\mathbf{r})\left(\varepsilon_{l}-\mu\right) \psi_{l \sigma}(\mathbf{r}) \\
& -\sum_{j, l} \int^{3} r \psi_{j \sigma}^{\dagger}(\mathbf{r}) \psi_{j \bar{\sigma}}^{\dagger}(\mathbf{r}) V_{j l} \psi_{l \bar{\sigma}}(\mathbf{r}) \psi_{l \sigma}(\mathbf{r}),
\end{aligned}
$$

where $\psi_{l \sigma}^{\dagger}\left(\psi_{l \sigma}\right)$ is the electron creation (annihilation) operator in the $l$-th band with the dispersion $\varepsilon_{l}(\mathbf{k})$ and the chemical potential $\mu$ and spin index $\sigma . V_{j l}$ is the intraband for $l=j$ and interband for $l \neq j$ scattering respectively, which can be either repulsive or attractive depending, for instance, on the strength of the Coulomb and electron-phonon interaction. The interband repulsion may cause frustration of the superconductivity in different bands and results in TRSB [6, 17]. Introducing the Nambu spinor operator $\Psi_{j}=\left(\psi_{j \uparrow}, \psi_{j \downarrow}^{\dagger}\right)^{T}$ and the energy gap $\Delta_{j}$ through the Hubbard-Stratonovich transform, we arrive at the following action in the imaginary time representation after integrating out the fermionic fields 23]

$$
S=\int d \tau d^{3} r \sum_{j, l}^{3} \Delta_{j} g_{j l} \Delta_{l}^{*}-\sum_{j} \operatorname{Tr} \ln \mathcal{G}_{j}^{-1}
$$

with $\hat{g}=\hat{V}^{-1}$ and the Gor'kov green function

$$
\mathcal{G}_{j}^{-1}=-\left(\begin{array}{cc}
\partial_{\tau}+\left(\varepsilon_{j}-\mu\right) & -\Delta_{j} \\
-\Delta_{j}^{*} & \partial_{\tau}-\left(\varepsilon_{j}-\mu\right)
\end{array}\right) .
$$

The superconducting energy gaps at $T=0$ are given by

$$
\sum_{l=1}^{3} \Delta_{l} g_{l j}=N_{j}(0) \Delta_{j} \sinh ^{-1}\left(\frac{\hbar \omega_{c j}}{\left|\Delta_{j}\right|}\right)
$$

with $N_{j}(0)$ the density of states (DOS) at the Fermi surface in normal state. Here $\omega_{c j}$ is a cutoff frequency and depends on the pairing mechanism. For electron-phonon coupling, $\omega_{c j}$ is the Debye frequency.

For demonstration of our basic idea, we take a set of simplified interband couplings [24]

$$
\hat{g}=\frac{1}{V}\left(\begin{array}{ccc}
\alpha & 1 & 1 \\
1 & \alpha & \eta \\
1 & \eta & \alpha
\end{array}\right)
$$

and assume that the DOS $N$ and $\omega_{c}$ are identical for three bands. 17] The massless LM, however, is not restricted to the specific choice of $\hat{g}$ as discussed later. Here $g_{i j}>0$ corresponds to a repulsive interaction. We take $\Delta_{1}$ as positive real, and $\Delta_{2}=\Delta e^{i \varphi}, \Delta_{3}=\Delta e^{-i \varphi}$ because they are symmetric under the condition of Eq. (5). Hereafter we take $\hbar \omega_{c}$ as the unit for $\Delta_{l}$.

For a small $\eta$, the interband repulsion $g_{12}$ and $g_{13}$ dominates and the system takes $\varphi=\pi$. For a large $\eta$, a state with finite phase difference between $\Delta_{2}$ and $\Delta_{3}$ appears, corresponding to
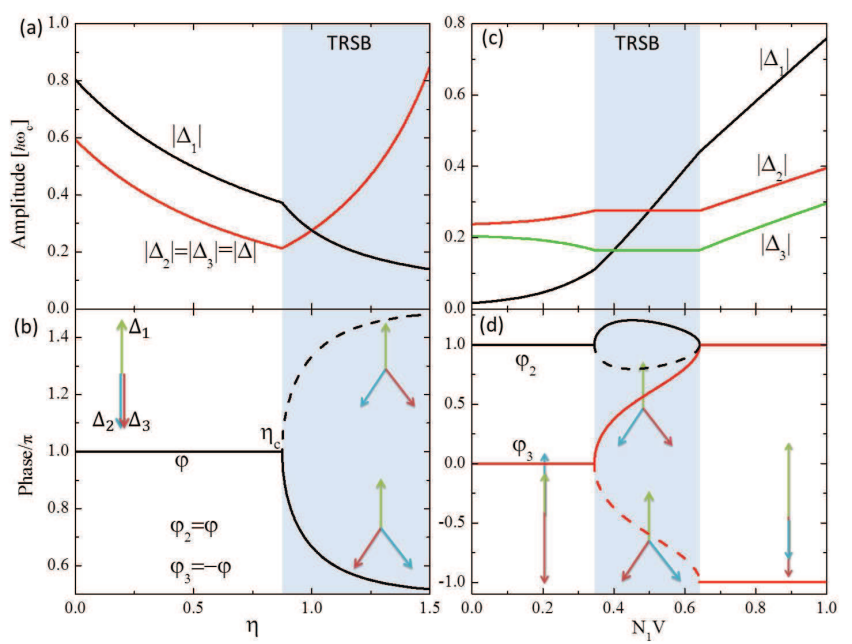

FIG. 2. (color online). Amplitudes and phases of order parameters at TRSB phase transition, in (a) and (b) as a function of $\eta$, and in (c, d) as a function of DOS $N_{1} V$ of the first component. $\Delta_{1}$ is taken as real and positive. In (a) and (b), an identical DOS $N V=0.5$ is taken for the three bands and $\alpha=2$ in Eq. (5). In (c) and (d), $N_{2} V=0.5$ and $N_{3} V=0.4, \alpha=2$ and $\eta=1$ (see also Eq. (17)). In the TRSB regime, there are two degenerate ground states $\left(\Delta_{1}, \Delta_{2}, \Delta_{3}\right)$ (solid lines) and $\left(\Delta_{1}^{*}, \Delta_{2}^{*}, \Delta_{3}^{*}\right)$ (dashed lines). The two solid lines for $N_{1} V>0.64$ in (d) refer to the same state without TRSB.

a state of TRSB where $\left(\Delta_{1}, \Delta_{2}, \Delta_{3}\right) \neq\left(\Delta_{1}, \Delta_{2}, \Delta_{3}\right)^{*}$, even apart the common phase factor. In the TRSB state the energy gaps are given by

$$
\Delta_{1}=1 / \sinh \left(\frac{\alpha \eta-1}{\eta} \frac{1}{N V}\right) \text {, and } \Delta=1 / \sinh \left(\frac{\alpha-\eta}{N V}\right)
$$

and $\cos \varphi=-\Delta_{1} /(2 \eta \Delta)$. The system undergoes a secondorder TRSB transition at $\eta_{c}$ given by $\eta_{c}=\Delta_{1}\left(\eta_{c}\right) /\left[2 \Delta\left(\eta_{c}\right)\right]$, as shown in Fig. 2 (a) and (b).

We proceed to investigate phase fluctuations at the TRSB point, where the amplitudes of the superconducting gap can be considered as rigid. For this purpose, we perform the following gauge transformation which separates the phase and amplitude of gap [11, 25]

$$
\Delta_{j} \rightarrow\left|\Delta_{j}\right| e^{i \theta_{j}} \text { and } \Psi_{j}(\tau, r) \rightarrow\left(\begin{array}{cc}
e^{i \theta_{j} / 2} & 0 \\
0 & e^{-i \theta_{j} / 2}
\end{array}\right) \Psi_{j}(\tau, r) .
$$

and derive the action for the phase fluctuation

$$
S=\int d \tau d^{3} r \sum_{j, l}\left|\Delta_{l}\right| g_{l j}\left|\Delta_{j}\right| e^{i\left(\theta_{l}-\theta_{j}\right)}-\sum_{j} \operatorname{Tr}\left[\ln \left(\mathcal{G}_{j}^{-1}-\Sigma_{j}\right)\right]
$$

where $\Sigma_{j}=-\frac{\hbar^{2}}{2 m_{j}}\left(\frac{i}{2} \nabla^{2} \theta_{j}+i \nabla \theta_{j} \nabla\right) \sigma_{0}+\left[i \frac{\partial_{\tau} \theta_{j}}{2}+\frac{\hbar^{2}}{8 m_{j}}\left(\nabla \theta_{j}\right)^{2}\right] \sigma_{3}$ with $\sigma_{j}$ being the Pauli matrices, $\sigma_{0}$ the unit matrix and $m_{j}$ the electron mass [26, 27]. From this action, one can obtain the time-dependent nonlinear Schrödinger Lagrangian for the phase fluctuations [28, 29]. Considering small phase fluctuations around the saddle point $\phi_{j}=\theta_{j}-\varphi_{j}$ and expanding $S$ up 
to the second order in $\phi_{j}$, we have 24]

$$
S_{\phi}\left[\phi_{j}\right]=\frac{1}{8} \sum_{l} \int d^{3} q \hat{\phi}\left(-\Omega_{l},-q\right)^{T} \mathbf{M} \hat{\phi}\left(\Omega_{l}, q\right)
$$

with $\hat{\phi}\left(\Omega_{l}, q\right) \equiv\left[\phi_{1}\left(\Omega_{l}, q\right), \phi_{2}\left(\Omega_{l}, q\right), \phi_{3}\left(\Omega_{l}, q\right)\right]^{T}$ and

$$
\mathbf{M}=\left(\begin{array}{ccc}
P_{1}-2 D_{1} & D_{1} & D_{1} \\
D_{1} & P_{2}-D_{1}-D_{2} & D_{2} \\
D_{1} & D_{2} & P_{3}-D_{1}-D_{2}
\end{array}\right)
$$

with $D_{1}=8 \Delta_{1} \Delta \cos \bar{\varphi} / V$ and $D_{2}=8 \eta \Delta^{2} \cos (2 \bar{\varphi}) / V$ with $\bar{\varphi} \equiv$ $\varphi_{2}-\varphi_{1}=\varphi_{1}-\varphi_{3} . \Omega_{l}=2 l \pi k_{B} T$ and the excitations are bosons. In the hydrodynamic limit at $T=0$, the dissipation is absent and $P_{j}=2 N\left(-\Omega^{2}+1 / 3 v_{j}^{2} q^{2}\right)$ after the analytical continuation $i \Omega_{l} \leftarrow \Omega+i 0^{+}$. From DetM $=0$, we obtain the dispersion relations

$$
\begin{aligned}
\Omega_{\mathrm{BAG}}^{2} & =\frac{1}{3} q^{2} v_{j}^{2}, \\
\Omega_{\mathrm{L}-}^{2} & =-\frac{D_{1}+2 D_{2}}{2 N}+\frac{1}{3} q^{2} v_{j}^{2}, \\
\Omega_{\mathrm{L}+}^{2} & =-\frac{3 D_{1}}{2 N}+\frac{1}{3} q^{2} v_{j}^{2} .
\end{aligned}
$$

The first mode is the massless BAG mode corresponding to the uniform rotation of phases. The second and third are the LM $\Omega_{\mathrm{L}-}$ and $\Omega_{\mathrm{L}+}$ in the present three-band system. Especially, the mode $\Omega_{\mathrm{L}}$ - corresponds to the dynamics of the relative phase $\varphi_{23}$ between the gaps of $\Delta_{2}$ and $\Delta_{3}$, and becomes massless at the TRSB transition depicted in Fig. 3 One may regard $\varphi_{23}$ as the order parameter for the TRSB transition. As it increases continuously from 0 at the transition, the associated fluctuations become massless at the TRSB transition.

The magnetic field can be introduced into $S_{\phi}$ through the standard replacement $\nabla \phi_{l} \rightarrow \nabla \phi_{l}-2 \pi \mathbf{A} / \Phi_{0}$ with $\Phi_{0}$ the flux quantum and $\mathbf{A}$ the vector potential. In this case, it is more convenient to describe the phase fluctuations in terms of $\phi_{1}$, $\phi_{12} \equiv \phi_{1}-\phi_{2}$ and $\phi_{13} \equiv \phi_{1}-\phi_{3} . \phi_{1}$ describes the BAG mode, and $\phi_{12}$ and $\phi_{13}$ correspond to the LMs. The gauge field couples with $\phi_{1}$ in the form $\left(\nabla \phi_{1}-2 \pi \mathbf{A} / \Phi_{0}\right)$. One may integrate out $\phi_{1}$, resulting in the massive plasma mode due to the Anderson-Higgs mechanism. In contrast to the BAG mode, the LMs remain massless at the TRSB transition since $\phi_{12}$ and $\phi_{13}$ are decoupled from the gauge field $\mathbf{A}$.

In stark contrast to conventional symmetry-broken systems, there exist the stable LMs both before and after TRSB transition, because the relative phase between different condensates is fixed in both the states with and without TRSB.

Raman scattering- Interband scatterings do not involve the gauge field, thus the LMs do not respond to a magnetic field. However, the LMs are coupled indirectly with the electric field through the charge density, which renders it detectable by the Raman spectroscopy through the inelastic scattering of photon with the charge density[30-33]. The interaction between the incident photon and the charge can be modeled as $\tilde{\rho}(\tau, q)=\sum_{j=1}^{3} \sum_{k, \sigma} \gamma_{j}(k) \psi_{j \sigma}^{\dagger}\left(\tau, k+\frac{q}{2}\right) \psi_{j \sigma}\left(\tau, k-\frac{q}{2}\right)$, where $\gamma_{j}(k)$

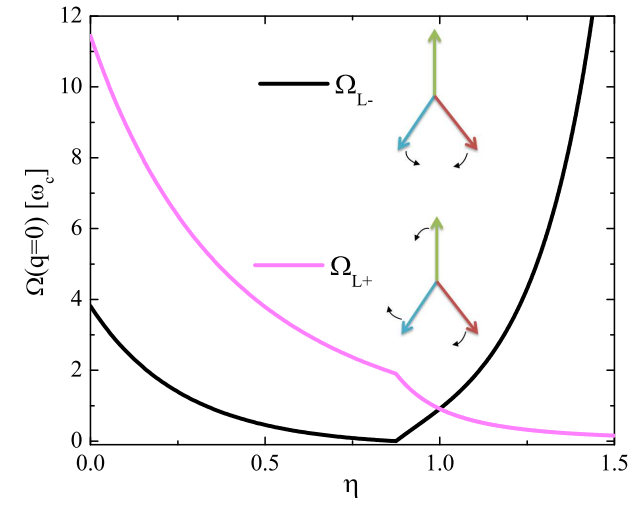

FIG. 3. (color online). Dependence of the masses of LMs on the interband coupling $\eta$. Here $N V=0.5$ and $\alpha=2$, and the masses are in units of $\omega_{c}$.

is the scattering coefficient determined by the polarization of the incident and scattered photon. In the following, we derive the experimentally measurable Raman response function $\chi_{\tilde{\rho} \tilde{\rho}}\left(\tau-\tau^{\prime}, q\right)=-\left\langle\mathrm{T}_{\tau} \tilde{\rho}(\tau, q) \tilde{\rho}\left(\tau^{\prime},-q\right)\right\rangle$ with $\mathrm{T}_{\tau}$ being timeordering operator. We introduce a source term coupled with $\tilde{\rho}, H_{J}(\tau)=-\sum_{q} \tilde{\rho}(\tau, q) J(\tau,-q)$ because $\chi_{\tilde{\rho} \tilde{\rho}}$ can be computed by the linear response theory with respect to $J$. The effective action in the presence of incident photon reads [24]

$$
S=\int d \tau d^{3} r \sum_{l, j} \Delta_{l} g_{l j} \Delta_{j}^{*}-\sum_{l} \operatorname{Tr} \ln \left(\mathcal{G}_{J, l}^{-1}+\mathcal{G}_{l}^{-1}\right)
$$

with $\mathcal{G}_{J, l}^{-1}=-\gamma_{l}(k) J(\tau,-q) \sigma_{3}$. For a weak incident wave, we may neglect the fluctuations of the amplitude of the order parameters, and the fluctuations for the superconducting phase acquires a form $S=S_{\phi}+S_{\mathrm{J}}$, with $S_{\phi}$ defined in Eq. (9) and

$$
\begin{aligned}
& S_{\mathrm{J}}=\frac{1}{2} \sum_{j, q}\left[J(q) Z_{j}(q) \phi_{j}^{T}(-q)\right. \\
& \left.+J(-q) \tilde{Z}_{j}(-q) \phi_{j}(q)+J(q) J(-q) \Pi_{j, 33}^{\gamma \gamma}\right],
\end{aligned}
$$

where $Z_{j}(q)=\Delta_{j}\left[-\sin \varphi_{j} \Pi_{j, 31}^{\gamma}(q)-\cos \varphi_{j} \Pi_{j, 32}^{\gamma}(q)\right]$ and $\tilde{Z}_{j}(q)=\Delta_{j}\left[-\sin \varphi_{j} \Pi_{j, 13}^{\gamma}(q)-\cos \varphi_{j} \Pi_{j, 23}^{\gamma}(q)\right] . \quad$ The polarization functions are defined as $\left[\Pi_{j, m l}^{\gamma \gamma}, \Pi_{j, m l}^{\gamma}\right] \equiv$ $1 /\left(L^{3} \beta\right) \sum_{n} \int d^{3} k \Upsilon_{j, m l}\left[\gamma_{j}\left(k+\frac{q}{2}\right) \gamma_{j}\left(k-\frac{q}{2}\right), \gamma_{j}\left(k+\frac{q}{2}\right)\right]$.

Integrating out the fluctuations $\phi_{j}$, we then obtain the correlation function

$$
\chi_{\tilde{\rho} \tilde{\rho}}(i \Omega, q=0)=\sum_{j}\left\{\Pi_{j, 33}^{\gamma \gamma}-Z_{j}\left[\mathbf{M}^{-1}\right]_{j j} \tilde{Z}_{j}^{T}\right\} .
$$

The first term gives the resonant scattering at $\Omega=2 \Delta_{j}$ and the second term accounts for the resonance with the LMs, as depicted in Fig. 4. When the energy shift of the photon matches the energy of the LMs, $\mathbf{M}^{-1}$ becomes singular and gives $\delta$ peaks in the spectroscopy. In reality, the delta-function peaks are rounded by both damping effect and interactions between Leggett bosons when the oscillations of the LM become 


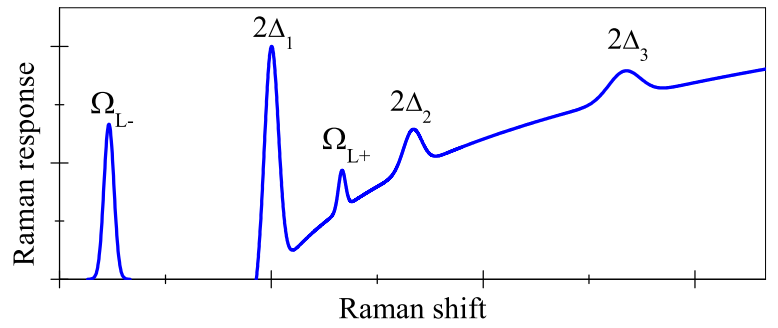

FIG. 4. (color online). Schematic view of the Raman response in three-band superconductors with TRSB. The finite line-width of peaks is due to damping and interaction between Leggett bosons. The background at energy larger than $2 \Delta_{1}$ is due to the quasiparticle excitations.

strong, which are absent in Eq. (16). Although the response of a genuinely massless LM is hidden into the elastic scatterings, it can be traced out clearly if one changes $\eta$ systematically and generates LM of small mass, which can be achieved by carrier doping because the interband scattering is renormalized by the DOS as in Eq. (4).

Discussions- At $T>0$, the Landau damping by quasiparticles sets in and the lifetime of the LM decreases. A local time-dependent equation for the phase fluctuations does not exist due to the singularity of the DOS in the superconducting state[34]. In the vicinity of $T_{c}$, the dynamics of superconductivity can be described by the standard time-dependent Ginzburg-Landau equation[34]. In this region, the lifetime of the LM is much smaller than the inverse of its energy due to the severe damping by quasiparticles, therefore there is no well-defined Leggett excitations. Nevertheless in the static case, the massless feature manifests as the divergence of the characteristic length for the relative phase variation in the vicinity of the TRSB [19].

Let us discuss the applicability of our results to the ironbased superconductors. In order to demonstrate the mass reduction of the LM by TRSB, we adopt a simple and general BCS-like Hamiltonian in Eq. (1). An implicit expectation behind this treatment is that more realistic models would merely lead to quantitative corrections. It seems that this simplification is not far from the situation in some iron-based superconductors, since the $s$-wave with sign-reversal ( $s \pm$ ) pairing symmetry is favored by many experiments [15, 16]. It also became clear recently that the interband hopping in single particle channels using a more realistic tight-binding model gives additional contribution to the interband Josephson coupling, and that the Hunds interaction only gives higher order correction to the LM[20]. It was also shown[35] that the $s \pm$ pairing can result from the moderate electronic correlations [36-40] in iron-based superconductors, thus electronic correlations probably do not hamper much the massless Leggett mode.

The reason that no direct experimental observation on the TRSB state in iron-pnictide superconductors has been reported to date may come from its requirement on sufficiently strong frustration interactions among different bands. Here we wish to observe that the TRSB transition can be induced not only by interband coupling but also by DOS $N_{j}(0)$. In order to demonstrate this we derive the TRSB solution to Eq. (4) under a general coupling matrix $\hat{g}$. Complex gap functions as solution to Eq. (4) appear when there is only one independent vector in the matrix $\hat{g}-\hat{g}^{\prime}$, with $g_{j j}^{\prime}=N_{j}(0) \sinh ^{-1}\left(\hbar \omega_{c j} /\left|\Delta_{j}\right|\right)$ and 0 otherwise. From this constraint we obtain[24]

$$
\frac{\left|\Delta_{j}\right|}{\hbar \omega_{c j}}=\frac{1}{\sinh \left[\left(g_{j j} g_{k l}-g_{j k} g_{j l}\right) / N_{j}(0) g_{k l}\right]},
$$

with $j \neq k \neq l$. It is easy to see that to find further the phases of the gap functions is equivalent to forming a triangle with the three segments $\left|\Delta_{j}\right| / g_{k l}$, which is possible when and only when $\left|\Delta_{j}\right| / g_{k l}+\left|\Delta_{k}\right| / g_{j l}>\left|\Delta_{l}\right| / g_{j k}$ for all the three combinations. The phase transition from a TRSB state to a state without TRSB takes place when one of the above inequalities is broken, for example $\left|\Delta_{1}\right| / g_{23}=\left|\Delta_{2}\right| / g_{13}+\left|\Delta_{3}\right| / g_{12}$. The results for DOSdriven TRSB transition are displayed in Fig. 2] (c) and (d). There are two TRSB transitions and the TRSB state is realized in a finite region of DOS. Therefore, experimentally one can tune $N_{j}(0)$ by careful chemical doping, which hopefully will drive the system to the TRSB transition.

Although the massless LM does not change magnetic properties of the system, it results in qualitatively different thermodynamic behaviors of $s$-wave superconductivity. For the $\mathrm{SH}$, the contribution due to quasiparticles at $T \ll T_{c}$ depends exponentially on temperature $\left(\Delta / k_{B} T\right)^{3 / 2} \exp \left(-\Delta / k_{B} T\right)$ for fully gapped superconductors. The contribution of the massless Leggett excitations can be obtained analytically by treating the Leggett bosons as free quantum gas. The contribution is of power-law temperature dependence $T^{3}$, which can be detected experimentally.

It is worth noting that a $T^{3}$ dependence of the $\mathrm{SH}$ in ironbase superconductors after subtracting the residue electronic contribution (linear in $T$ ) and phonon contribution (also $T^{3}$ dependence) has been reported in several experiments, [4143]; fully gapped order parameters are inferred from measurements for the dependence of electronic SH on magnetic field, which excluded the possibility of gap function of line node. Actually, in Ref. [42], the authors suggested that the additional $T^{3}$ contribution might be due to some bosonic modes. These experimental observations can be naturally explained by the existence massless LM. Additional measurements such as the Raman spectroscopy on similar samples [41-43] are much anticipated which may well be in the vicinity of the TRSB transition.

Acknowledgements - The authors are grateful for L. Bulaevskii, J. -X. Zhu and Z. Wang for discussions. This work was supported by WPI Initiative on Materials Nanoarchitectonics, and Grants-in-Aid for Scientific Research (No.22540377), MEXT, Japan, and partially by CREST, JST. 
[1] P. W. Anderson, Phys. Rev. 110, 827 (1958).

[2] N. N. Bogoliubov, Sov. Phys. Usp. 673, 236 (1959).

[3] J. Nagamatsu, N. Nakagawa, T. Muranaka, Y. Zenitani, and J. Akimitsu, Nature 410, 63 (2001).

[4] Y. Kamihara, T. Watanabe, M. Hirano, and H. Hosono, J. Am. Chem. Soc. 130, 3296 (2008).

[5] A. J. Leggett, Prog. Theor. Phys. 36, 901 (1966).

[6] D. F. Agterberg, V. Barzykin, and L. P. Gor'kov, Phys. Rev. B 60, 14868 (1999).

[7] Y. Tanaka, Phys. Rev. Lett. 88, 017002 (2002).

[8] E. Babaev, Phys. Rev. Lett. 89, 067001 (2002).

[9] G. Blumberg, A. Mialitsin, B. S. Dennis, M. V. Klein, N. D. Zhigadlo, and J. Karpinski, Phys. Rev. Lett. 99, 227002 (2007).

[10] X. X. Xi, Rep. Prog. Phys. 71, 116501 (2008).

[11] S. C. Sharapov, V. P. Gusynin, and H. Beck, Eur. Phys. J. B 30, 45 (2002).

[12] Y. G. Ponomarev, S. A. Kuzmicheva, M. G. Mikheev, M. V. Sudakova, S. N. Tchesnokov, N. Z. Timergaleev, A. Yarigin, E. G. Maksimov, S. I. Krasnosvobodtsev, A. Varlashkin, M. A. Hein, G. Muller, H. Piel, L. G. Sevastyanova, O. Kravchenko, K. P. Burdina, and B. M. Bulychev, Solid State Commun. 129, 85 (2004).

[13] I. I. Mazin, D. J. Singh, M. D. Johannes, and M. H. Du, Phys. Rev. Lett. 101, 057003 (2008).

[14] K. Kuroki, S. Onari, R. Arita, H. Usui, Y. Tanaka, H. Kontani, and H. Aoki, Phys. Rev. Lett. 101, 087004 (2008).

[15] H. Ding, P. Richard, K. Nakayama, K. Sugawara, T. Arakane, Y. Sekiba, A. Takayama, S. Souma, T. Sato, T. Takahashi, Z. Wang, X. Dai, Z. Fang, G. F. Chen, J. L. Luo, and N. L. Wang, Europhys. Lett. 83, 47001 (2008).

[16] C. T. Chen, C. C. Tsuei, M. B. Ketchen, Z. A. Ren, and Z. X. Zhao, Nat. Phys. 6, 260 (2010).

[17] V. Stanev and Z. Tešanović, Phys. Rev. B 81, 134522 (2010).

[18] Y. Tanaka and T. Yanagisawa, J. Phys. Soc. Jpn. 79, 114706 (2010).

[19] X. Hu and Z. Wang, arXiv:1103.0123 (2011).

[20] F. J. Burnell, J. P. Hu, M. M. Parish, and B. A. Bernevig, Phys. Rev. B 82, 144506 (2010).

[21] Y. Ota, M. Machida, T. Koyama, and H. Aoki, Phys. Rev. B 83, 060507 (2011).

[22] A. V. Balatsky, P. Kumar, and J. R. Schrieffer, Phys. Rev. Lett.
84, 4445 (2000).

[23] A. Alexander and B. D. Simons, Condensed Matter Field Theory (Cambridge University Press, Cambridge, 2010).

[24] See the EPAPS Document No. xxx for details of derivations. For more information on EPAPS, see http://www.aip.org/pubservs/epaps.html

[25] V. M. Loktev, R. M. Quick, and S. G. Sharapov, Phys. Rep. 349, 1 (2001).

[26] S. De Palo, C. Castellani, C. Di Castro, and B. K. Chakraverty, Phys. Rev. B 60, 564 (1999).

[27] L. Benfatto, A. Toschi, and S. Caprara, Phys. Rev. B 69, 184510 (2004).

[28] I. J. R. Aitchison, P. Ao, D. J. Thouless, and X. M. Zhu, Phys. Rev. B 51, 6531 (1995).

[29] I. J. R. Aitchison, G. Metikas, and D. J. Lee, Phys. Rev. B 62, 6638 (2000).

[30] A. A. Abrikosov and L. A. Falkovskii, Sov. Phys. JETP 13, 179 (1961).

[31] M. V. Klein and S. B. Dierker, Phys. Rev. B 29, 4976 (1984).

[32] T. P. Devereaux and D. Einzel, Phys. Rev. B 51, 16336 (1995).

[33] H. C. Lee and H. Y. Choi, J. Phys.: Condens. Matter 21, 445701 (2009).

[34] E. Abrahams and T. Tsuneto, Phys. Rev. 152, 416 (1966).

[35] S. Graser, T. A. Maier, P. J. Hirschfeld, and D. J. Scalapino, New J. Phys. 11, 025016 (2009).

[36] M. M. Qazilbash, J. J. Hamlin, R. E. Baumbach, L. Zhang, D. J. Singh, M. B. Maple, and D. N. Basov, Nat. Phys. 5, 647 (2009).

[37] K. Haule and G. Kotliar, New J. Phys. 11, 025021 (2009).

[38] M. Aichhorn, L. Pourovskii, V. Vildosola, M. Ferrero, O. Parcollet, T. Miyake, A. Georges, and S. Biermann, Phys. Rev. B 80, 085101 (2009).

[39] Q. Si, E. Abrahams, J. Dai, and J.-X. Zhu, New J. Phys. 11, 045001 (2009).

[40] P. Hansmann, R. Arita, A. Toschi, S. Sakai, G. Sangiovanni, and K. Held, Phys. Rev. Lett. 104, 197002 (2010).

[41] J. S. Kim, P. J. Hirschfeld, G. R. Stewart, S. Kasahara, T. Shibauchi, T. Terashima, and Y. Matsuda, Phys. Rev. B 81, 214507 (2010).

[42] K. Gofryk, A. B. Vorontsov, I. Vekhter, A. S. Sefat, T. Imai, E. D. Bauer, J. D. Thompson, and F. Ronning, Phys. Rev. B 83, 064513 (2011).

[43] B. Zeng, B. Shen, G. F. Chen, J. B. He, D. M. Wang, C. H. Li, and H. H. Wen, Phys. Rev. B 83, 144511 (2011). 